\title{
Signature of the State measures on the COVID-19 Pandemic in China, Italy, and USA
}

\author{
Farhan Saif ${ }^{1}$ \\ ${ }^{1}$ Department of Electronics, Quaid-i-Azam University, 45320, Islamabad, Pakistan.
}

( Dated: April 8, 2020)

\begin{abstract}
We show the dynamics of COVID-19 outbreak in Italy and USA, in comparison with China, and the early response of the countries. Our mathematical techniques makes it possible to calculate the rate of growth of the cases efficiently, and provides a good understanding of future trends in Italy and USA. The evolution of the real time data makes it possible to analyse the suitability of steps taken to eradicate the pandemic by the countries. We compare the day to day development of the coronavirus cases in Italy and USA, that keeping in view the population pyramid and the population density, leads us to understand possible difference in the number of effected population.
\end{abstract}

a. Introduction On December 27, 2019, a hospital in Wuhan, capital of Hubei province, reported the first mysterious, suspected pneumonia cases to the center of disease control (CDC) in the capital. On January 8, 2020 a new coronavirus was identified as the cause of pneumonia that spread all over China in next three weeks. Since then, the novel coronavirus, COVID-19, spread across the globe in the next month. In March the World Health Organization declared it to be a pandemic, that in the absence of a vaccine became a challenge for mankind with a tendency to grow exponentially if no measure is taken to prevent it [? ]. The incubation period of COVID-19 can last for two weeks or longer [2]. During the period of latent infection, the disease may still be infectious [3]. The virus can spread from person to person through respiratory droplets and close contact [4].

Employing social distancing, following patient history case by case, and locking down the province of Hubei, and de-facto quarantining the other provinces by restricting the movement of people China controlled the COVID19 spread in thirty five days successfully with minimum number of deaths. On the other hand similar out break in Iran and Italy remained uncontrolled after forty five and sixty days, respectively, with forty five thousands in Iran and one hundred and five thousands people in Italy tested positive as on March 31. In this paper based on mathematical modelling and reported previous evolution in China, we predict the future dynamics of the COVID19 outbreak in the second quarter of 2020 in Italy and USA. The lack of testing facilities and sanctions are restricting the authenticity of data from Iran, for the reason we are not including it in the present publication. For our study related to China we have considered the first twenty one days data that is from January 16, till February 05 , whereas we have excluded the epicentre province of Hubei, which had a significant issue of under reporting at the early stage and also data inconsistency due to a change of classification guidelines. For Italy and USA we have considered the data from February 01, till April 06.

Various mathematical model to analyse the growth of an epidemic are employed, that include SEIR model [5], generalized SEIR model [6] and neural network based quarantine control model [7]. Moreover mathematical analysis of pandemics based on exponential growth model [8-11] and generalized growth model [12-22] have been reported. In this contribution, we have developed discrete generalized growth model $\left(\mathrm{DG}^{2} \mathrm{M}\right)$, the discrete generalized logistic model (DGLM), and discrete generalized Richard model (DGRM). These models have their limitations and are only applicable to some stages, however they provide more accurate measure of the growth in the outbreak. Based on our numerical results we find excellent agreement between the analytical results and the real-time data of coronavirus positively tested cases. The top-down methods have the advantage to study future scenario of the outbreak, provide lower and upper bounds of our predictions, the rate of growth in stages and provide informative implications of the measures taken to control and eradicate the pandemic.

The manuscript has the following layout: in section II we present the mathematical model for our study, in section III we explain the real-time data of positively tested COVID-19 patients with the numerical results based on our models. In section IV we present our estimates and recommendations based on the work.

b. Mathematical Model The generalized Richard Model [23], that is

$$
\frac{d C(t)}{d t}=r C(t)^{p}\left(1-\left(\frac{C(t)}{K}\right)^{\alpha}\right) .
$$

is used in explaining previous epidemics. It is the extension of the original Richards growth model [24] which explain the pandemics [25-29]. Here, $r$ is the growth rate at the early stage, and $K$ is the final epidemic size. Moreover $p$ is a parameter that allows the model to capture different growth profiles. The exponent $\alpha$ measures the deviation from the dynamics of the simple logistic curve. The equation (1) corresponds to the original Richards model for $p=1$, that is

$$
\frac{d C(t)}{d t}=r C(t)\left(1-\left(\frac{C(t)}{K}\right)^{\alpha}\right),
$$

and reduces to the generalized logistic model for $\alpha=1$ and $p=1$.

Keeping in view the development of data in terms of the positive tests, variations, and deaths on every next day we may consider the change in $C$ over a unit interval of time. We express the rate of change of $C$, that is $\dot{C}(t)$, 
in difference form as $\frac{\Delta C}{\Delta t}$. Here $\Delta C=C_{n+1}-C_{n}$ is the change in positively tested patients. Thus we reshape the equation (1) in the discrete form as

$$
C_{n+1}=C_{n}+r C_{n}^{p}\left(1-\left(\frac{C_{n}}{K}\right)^{\alpha}\right),
$$

where $C_{n}$ is the number of positively tested patients on $n$th day. The discrete generalized Richard model (DGRM) provides an effective way to monitor the pandemic in the form of a mapping, thus connecting the data on $n$th day with $(n+1)$ th day. The stable points of the discrete generalized Richards model given in equation (3) are $C_{n}=0$, that implies no positively tested patients, and $C_{n}=K$. The stable points are the same as obtained by the generalized Richards model.

c. Numerical results and real-time data We write the discrete generalized growth model $\left(\mathrm{DG}^{2} \mathrm{M}\right)$, as

$$
C_{n+1}=C_{n}+r C_{n}^{p}
$$

which provides exponential growth for $p=1$, that is $C_{n+1}=r_{1}^{n} C_{1}$, that relates the number of positively tested patients on the first day with the number of postively tested patients after a number of $n$ days has passed, here $r_{1}=1+r$. Hence for the parameter $p=0$ we find linear increase with respect to $n$ as $C_{n+1}=C_{1}+n r$, and sub-exponential growth for $0<p<1$.

The outbreak is discussed in the initial phase in the three countries, that is China, Italy and USA. In the presence of an almost consistent growth of young population and large old population, the population pyramid in the three countries has close resemblance, as shown in figure ??. The population between 15 and 65 years is $70 \%, 64 \%$ and $63 \%$, whereas above 65 years of age in China, Italy and USA is, respectively, 9.55\%, $22.41 \%$ and $14.79 \%$. However the pandemic has effected the three countries in diversely different pattern hinting at the preparedness of the countries to tackle such natural disasters as we show in the following discussion.

In order to make a relation between the number of positively tested cases reported every day with the increase in the number of these cases per day, we develop double $\log$ plot, shown in figure 1 . We compare the data of the patients with the discrete generalized growth model given in equation (4). Taking the logrythm of the equation (4), we write the corresponding relation for the logrythm of the increase in the number of patients $\log \Delta C_{n}$ as

$$
\log \Delta C_{n}=p \log C_{n}+\log r,
$$

which is the equation of a straight line, in $\left(\log \Delta C_{n}, \log C_{n}\right)$ plane. Here $p$ expresses the slope of the straight line.

Interestingly a comparison of the real time data of the positively tested patients with equation (5) makes it appear in two sub-groups: First sub-group of the data makes a straight line that describes the evolution of COVID-19 pandemic in its early days. The outbreak follows a certain rate $r$ of its spread that define the corresponding straight line as expressed in equation (5). The
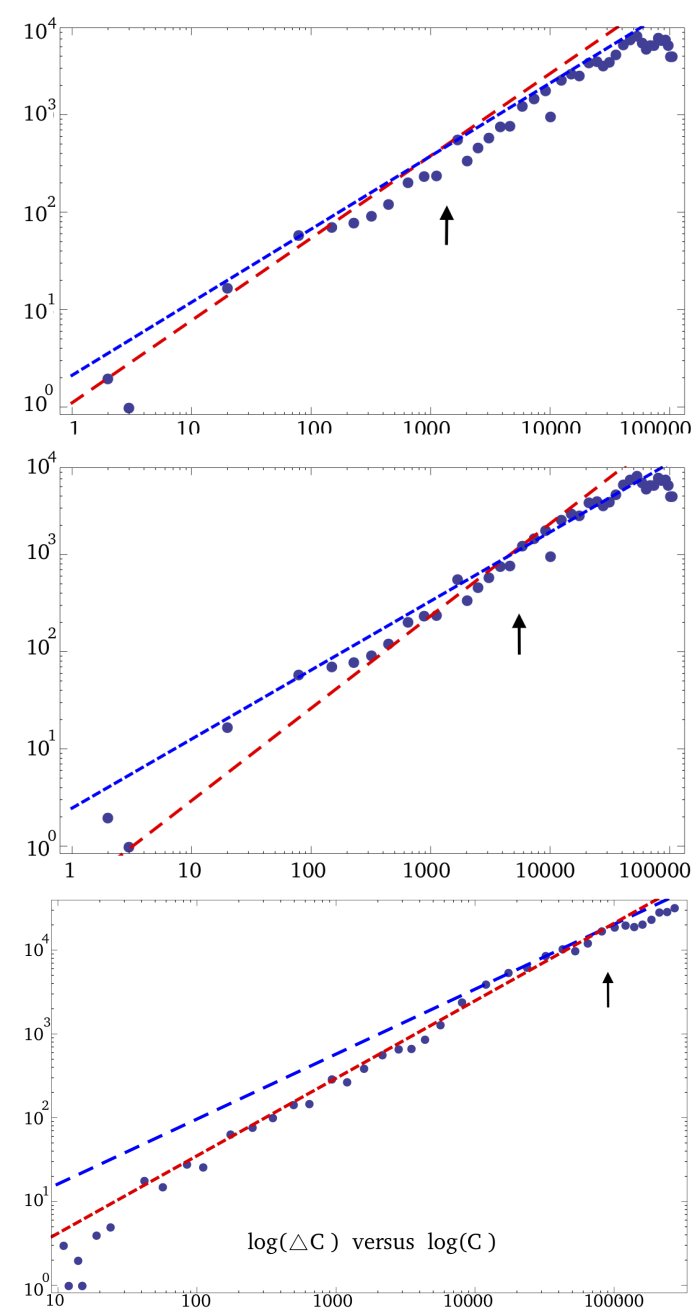

FIG. 1. We show log-log plot between the number of positive cases and the day-to-day increase in number in the alphabetical order: In the upper panel we show the real-time data of patients in China showing logrythm of the number of increase in the positively tested COVID-19 patients as a function of logrythm of positively tested patients. We compare mathematical plots obtained by $\mathrm{DG}^{2} \mathrm{M}$ shown in red dashed-line for $r=1.105$ and $p=0.845$, whereas the blue dashed-line is plotted by using DGRM for $r=2.117, p=0.75, \alpha=1.3$, and $K=60,000$. In the middle panel we show real-time data for Italy. We compare the mathematical plots obtained by $\mathrm{DG}^{2} \mathrm{M}$ model shown in red dashed-line for $r=2.46$ and $p=0.72$, whereas the line plotted by means of DGRM has $r=0.33$, $p=0.95, \alpha=1$, and $K=155,000$; In the lower panel we show the comparison for USA. Here we have the data obtained by $\mathrm{DG}^{2} \mathrm{M}$ shown in red dashed-line for $r=0.202$ and $p=1.115$, whereas the blue dashed-line plotted by employing DGRM for $r=2.718, p=0.775, \alpha=1.4$, and $K=950,000$.

parameter $p$ describes the spread of the outbreak, it is sub-exponential for $0<p<1$, exponential for $p=1$, and faster than exponential for $p>1$. It appears in figure 1 as a red line for the three different countries, that is China, Italy and USA respectively; Second, the next stage of the outbreak makes another straight line as shown by blue 
line in figure 1. The point of intersection of the two lines, indicated by vertical arrows in figure 1, corresponds to the preventive measures adopted by the respective country. For example, the early intersection of the two lines in case of China indicates a general lock down in most of the provinces of China on January 26,2020, as soon as the reported cases were around one thousands. Whereas in the case of Italy it happened after an order of magnitude increase in number that is around 10000, as appears in figure 1 . In case of USA we find the intersection point appears even farther.

We obtain the mathematical results by the discrete generalized growth model, discrete generalized logistic growth model and the discrete generalized Richard model by using the $r$ and $p$ values obtained from above discussion for China, Italy and USA. The obtained results are compared with the real time data of COVID-19 patients obtained from these countries, as shown in figure 2.

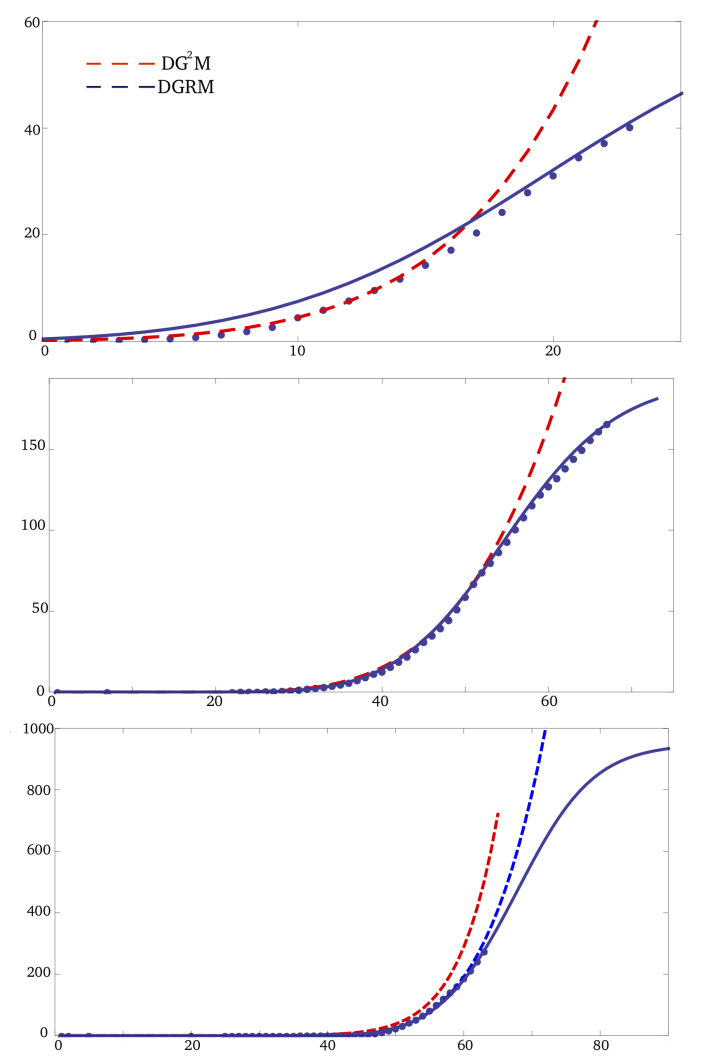

FIG. 2. We plot the number (in thousands) of positively tested cases versus the number of days, in the alphabetical order for China, Italy and USA. The description of the red dashed-line and blue dashed-line, and the values of the parameters is the same as given in figure 1. The blue dashed line in lower panel dedicated to USA data shows the pandemic dynamics following $D^{2} \mathrm{M}$, whereas the blue line expresses the COVID-19 pandemic dynamics following DGRM.

The plots show that the discrete generalized growth model $\left(D^{2} \mathrm{M}\right)$ show the gradual increase that matches very good with the real time data of positively tested patients in the three countries. The preventive measures taken in China show a gradual deviation from the $\mathrm{DG}^{2} \mathrm{M}$ model, whereas it is taken over by the DGRM. In case of Italy the gradual deviation of real-time data is also seen, reflecting a slow down of the outbreak, that indicates a positive effect of the steps taken by the government of Italy, such as strict lock down, intercity travel and closure of education sector, and unnecessary travels. In case of USA, the data is showing a deviation from $\mathrm{DG}^{2} \mathrm{M}$, however it is not significantly close of DGRM. The blue dashed-line indicates the future growth of the coronavirus pandemic following $\mathrm{DG}^{2} \mathrm{M}$ model showing an uncontrolled increase. Whereas the blue line shows the future trend of the pandemic following DGRM model. This implies that to eradicate the COVID-19 successfully it is required to take further strict measures.

The logrhythmic evolution, in figure 3, expresses the size of the outbreak in China, Italy and USA. As the estimated size of outbreak agrees well with the reported data of China and Italy, it is expected that in USA in the absence of further strict measures the coronavirus pandemic may effect around a million people in next fifteen days, almost $0.3 \%$ of USA population in another five days and before we see a slowing down effect.

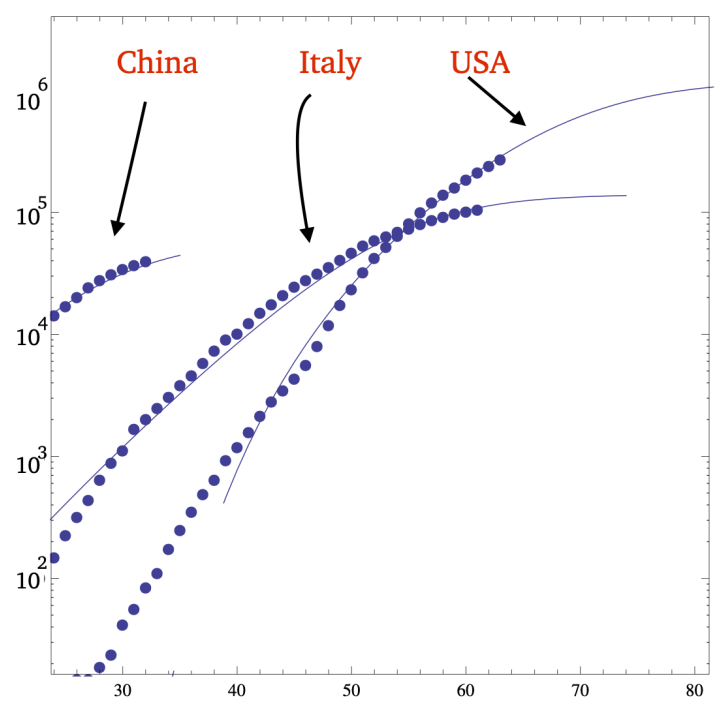

FIG. 3. Along the vertical axis we show the logrythm of number of positively tested cases of COVID-19, and the number of days are along the horizontal axis. The blue dashed-lines are obtained by DGRM whereas the blue dots represent the real-time data. The values of the parameters are the same as given in figure 1.

d. Conclusions We show that an early response from Italy and USA was necessary to restrict the wide spread of the pandemic. In comparison with the COVID19 early evolution in China we provide quantitative analysis of the outbreak in Italy and USA. We mathematically identify the transition in the evolution of the pandemic as a function of measure taken by respective governments by classification of corresponding data in stages which are defined by mathematical parameters. We pro- 
vide rate of growth at every stage, and measure of the nature of expansion of pandemic. We predict a high risk in USA, where the number of positively tested coronavirus cases may reach one million in the absence of more strict measures in next fifteen days, and in next twenty days $0.3 \%$ USA population may get infected in a positive [who] oint Mission WHO. Report of the who-china joint mission on coronavirus disease 2019 (covid19), 2020. URL https://www.who.int/docs/defaultsource/coronaviruse/ who-china-joint-mission-on-covid19-final-report.pdf. (accessed March 28, 2020).

[2] World Health Organization, "Report of the WHO-China Joint Mission on Coronavirus Disease 2019 (COVID19)," World Health Organization, 2020.

[3] Ruiyun Li, Sen Pei, Bin Chen, Yimeng Song, Tao Zhang, Wan Yang, and Jeffrey Shaman. Substantial undocumented infection facilitates the rapid dissemination of novel coronavirus (sars-cov2). Science, 2020.

[4] "Coronavirus Disease 2019 (COVID-19)," Centers for Disease Control and Prevention, 2020. [Online]. Available: https://www.cdc.gov/coronavirus/2019ncov/about/symptoms.html. [Accessed 27 February 2020].

[5] Binti Hamzah FA, Lau C, Nazri H, Ligot DV, Lee G, Tan CL, et al. CoronaTracker: World- wide COVID19 Outbreak Data Analysis and Prediction. [Submitted]. Bull World Health Organ. E-pub: 19 March 2020. doi: http://dx.doi.org/10.2471/BLT.20.255695

[6] Lenka Pribiylova and Veronika Hajnova, SEIAR model with asymptomatic cohort and consequences to efficiency of quarantine government measures in COVID-19 outbreak, arXiv:2004.02601-v1

[7] Raj Dandekar and George Barbastathis, Neural Network aided quarantine control model estimation of global Covid-19 spread, arXiv:2004.02752v1

[8] Tuite AR, Fisman DN. Reporting, Epidemic Growth, and Reproduction Numbers for the 2019 Novel Coronavirus (2019-nCoV) Epidemic. Ann Intern Med. 2020.(February):2019-20.

[9] Zhao S, Cao P, Gao D, Zhuang Z, Chong MKC, Cai Y. Epidemic growth and reproduction number for the novel coronavirus disease ( COVID-19) outbreak on the Diamond Princess cruise ship from January 20 to February 19 , 2020 : A preliminary data- driven analysis. SSRN. 2020. Preprint at: https://ssrn.com/abstract $=3543150$

[10] You C, Deng Y, Hu W, Sun J, Lin Q, Zhou F, et al. Estimation of the Time-Varying Reproduction Number of COVID-19 Outbreak in China.

[11] Zhang S, Diao M, Yu W, Pei L, Lin Z, Chen D. Estimation of the reproductive number of Novel Coronavirus (COVID-19) and the probable outbreak size on the Diamond Princess cruise ship: A data-driven analysis. Int J Infect Dis. 2020.

[12] Li Y, Yin X, Liang M, Liu X, Hao M, Wang Y. A Note on NCP Diagnosis Number Prediction Model. medRxiv. 2020. Preprint at: https://www.medrxiv.org/content /10.1101/2020.02.19.20025262v1

[13] Maier BF, Brockmann D. Effective containment explains sub-exponential growth in confirmed cases of recent COVID-19 outbreak in Mainland China. arXiv. 2020. Preprint at: https://arxiv.org/abs/2002.07572
[14] Ying S, Li F, Geng X, Li Z, Du X, Chen H, et al. Spread and control of COVID-19 in China and their associations with population movement, public health emergency measures, and medical resources. medRxiv. 2020. Preprint at: https://www.medrxiv.org/content /10.1101/2020.02.24.20027623v1

[15] Brandenburg A. Quadratic growth during the 2019 novel coronavirus epidemic. arXiv. 2020. Preprint at: http://arxiv.org/abs/2002.03638

[16] Ziff AL, Ziff RM. Fractal kinetics of COVID-19 pandemic. medRxiv. 2020. Preprint at: https://www.medrxiv.org/content /10.1101/2020.02.16.20023820v2

[17] Muniz-Rodriguez K, Chowell G, Cheung C-H, Jia D, Lai P-Y, Lee Y, et al. Epidemic doubling time of the COVID-19 epidemic by Chinese province. medRxiv. 2020. Preprint at: https://www.medrxiv.org/content $/ 10.1101 / 2020.02 .05 .20020750 \mathrm{v} 4$

[18] Zhang J, Litvinova M, Wang W, Wang Y, Deng $\mathrm{X}$, Chen $\mathrm{X}$, et al. Evolving epidemiology of novel coronavirus diseases 2019 and possible interruption of local transmission outside Hubei Province in China: a descriptive and modeling study. medRxiv. 2020. Preprint at: https://www.medrxiv.org/content /10.1101/2020.02.21.20026328v1

[19] Roosa K, Lee Y, Luo R, Kirpich A, Rothenberg R, Hyman JM, et al. Real-time forecasts of the 2019-nCoV epidemic in China from February 5th to February 24th, 2020. Infect Dis Model. 2020. 40

[20] Roosa K, Lee Y, Luo R, Kirpich A, Rothenberg R, Hyman JM, et al. Short-term Forecasts of the COVID-19 Epidemic in Guangdong and Zhejiang, China: February 13-23, 2020. J Clin Med. 2020.9(2):596.

[21] Wu K, Zheng J, Chen J. Utilize State Transition Matrix Model to Predict the Novel Corona Virus Infection Peak and Patient Distribution. SSRN. 2020. Preprint at: https://papers.ssrn.com/sol3/papers.cfm? abstract $\mathrm{id}=3539658$

[22] Lin H, Liu W, Gao H, Nie J, Fan Q. Trends in Transmissibility of 2019 Novel Coronavirus-infected Pneumonia in Wuhan and 29 Provinces in China. SSRN. 2020. Preprint at: https://papers.ssrn.com/sol3/papers.cfm?abstract id $=3544821$

[23] $\mathrm{Ke} \mathrm{Wu}$ and Didier Darcet and Qian Wang and Didier Sornette, Generalized logistic growth modeling of the COVID-19 outbreak in 29 provinces in China and in the rest of the world, arXiv:2003.05681.

[24] Richards FJ. A flexible growth function for empirical use. J Exp Bot. 1959.10(2):290- 301.

[25] Chowell G. Fitting dynamic models to epidemic outbreaks with quantified uncertainty: A primer for parameter uncertainty, identifiability, and forecasts. Infect Dis Model. 2017.2(3):379-98.

[26] Viboud C, Simonsen L, Chowell G. A generalized-growth model to characterize the early ascending phase of infec- 
medRxiv preprint doi: https://doi.org/10.1101/2020.04.08.20057489; this version posted April 10, 2020. The copyright holder for this preprint (which was not certified by peer review) is the author/funder, who has granted medRxiv a license to display the preprint in perpetuity.

It is made available under a CC-BY 4.0 International license.

tious disease outbreaks. Epidemics. 2016.15:27-37.

[27] Chowell G, Tariq A, Hyman JM. A novel sub-epidemic modeling framework for short- term forecasting epidemic waves. BMC Med. 2019.17(1):1-18.

[28] Chowell G, Hincapie-Palacio D, Ospina J, Pell B, Tariq A, Dahal S, et al. Using Phenomenological Models to Characterize Transmissibility and Forecast Patterns and
Final Burden of Zika Epidemics. PLoS Curr. 2016.8.

[29] Chowell G, Luo R, Sun K, Roosa K, Tariq A, Viboud C. Real-time forecasting of epidemic trajectories using computational dynamic ensembles. Epidemics. 2020.30(November 2019):100379. 